\title{
Melioidosis in a Resident of Texas with No Recent Travel History, United States
}

Caitlin M. Cossaboom, Atanaska Marinova-Petkova, Jonathan Strysko, Gretchen Rodriguez, Trevor Maness, Jaime Ocampo, Jay E. Gee, Mindy G. Elrod, Christopher A. Gulvik, Lindy Liu, William A. Bower, Alex R. Hoffmaster, David D. Blaney, Johanna S. Salzer, Jonathan S. Yoder, Mia C. Mattioli, Thomas J. Sidwa, Lillian Ringsdorf, Gale Morrow, Elvia Ledezma, Amanda Kieffer

To our knowledge, environmental isolation of Burkholderia pseudomallei, the causative agent of melioidosis, from the continental United States has not been reported. We report a case of melioidosis in a Texas resident. Genomic analysis indicated that the isolate groups with $B$. pseudomallei isolates from patients in the same region, suggesting possible endemicity to this region.

$B$ urkholderia pseudomallei, which causes melioidosis, is a gram-negative saprophytic bacterium endemic to tropical and subtropical environments worldwide; to our knowledge, isolation from the continental United States has not been reported (1$3)$. The most overrepresented risk factor for melioidosis is diabetes mellitus $(3,4)$. B. pseudomallei is resistant to many antimicrobial drugs (3). Laboratory exposures might occur without appropriate biosafety precautions $(2,5)$.

Surveillance is challenging because melioidosis is not nationally notifiable; however, B. pseudomallei is a Tier 1 overlap Select Agent (6), and the Centers for Disease Control and Prevention (CDC) receives voluntary reports $(2,7)$. We report a case of melioidosis in a Texas resident who had no recent travel history.

Author affiliations: Centers for Disease Control and Prevention,

Atlanta, Georgia, USA (C.M. Cossaboom, A. Marinova-Petkova,

J. Strysko, J.E. Gee, M.G. Elrod, C.A. Gulvik, L. Liu, W.A.

Bower, A.R. Hoffmaster, D.D. Blaney, J.S. Salzer, J.S. Yoder, M.C.Mattioli); Texas Department of State Health Services,

San Antonio, Texas, USA (G. Rodriguez, T. Maness, J. Ocampo,

L. Ringsdorf, G. Morrow, E. Ledezma, A. Kieffer); Texas

Department of State Health Services, Austin, Texas, USA

(T.J. Sidwa)

DOI: https://doi.org/10.3201/eid2606.190975

\section{The Study}

On November 17, 2018, a 63-year-old man from Atascosa County, Texas, came to hospital A with fever, chest pain, and dyspnea of 3 days' duration. At admission, he reported congenital unilateral renal agenesis. Renal function measures were unremarkable. Increased hemoglobin A1c level and hyperglycemia (glucose $>200 \mathrm{mg} / \mathrm{dL}$ ) suggested undiagnosed type 2 diabetes.

Computed tomography of chest and abdomen with intravenous contrast showed left lower lobe pneumonia with a small left pleural effusion. Empiric antimicrobial drug therapy with intravenous azithromycin and ceftriaxone was initiated. Blood culture yielded presumptive B. pseudomallei, which was sent for confirmation to the laboratory response network (LRN) site in Houston, Texas.

On November 20, a localized, violaceous, cutaneous lesion developed on the central anterior chest wall of the patient and progressed to become purulent and ulcerated (Figure 1). The next day, he experienced respiratory failure, was emergently intubated, and was transferred to hospital B.

Hospital B was not aware of the presumptive diagnosis and performed a blood and chest wound culture. Both cultures showed gram-negative rods; blood analysis showed acute kidney injury. On November 25 , B. pseudomallei susceptible to trimethoprim/sulfamethoxazole and ceftazidime (Table) was identified, and treatment was switched to ceftazidime by using dosing for continuous renal replacement therapy (4).

On November 26, the patient was extubated and began hemodialysis $(3 \times / w k)$. He was discharged on December 9 and received 3 months of daily trimethoprim/sulfamethoxazole (4). Subsequent followup showed clinically recovery and resolution of $\mathrm{z}$ renal insufficiency. 


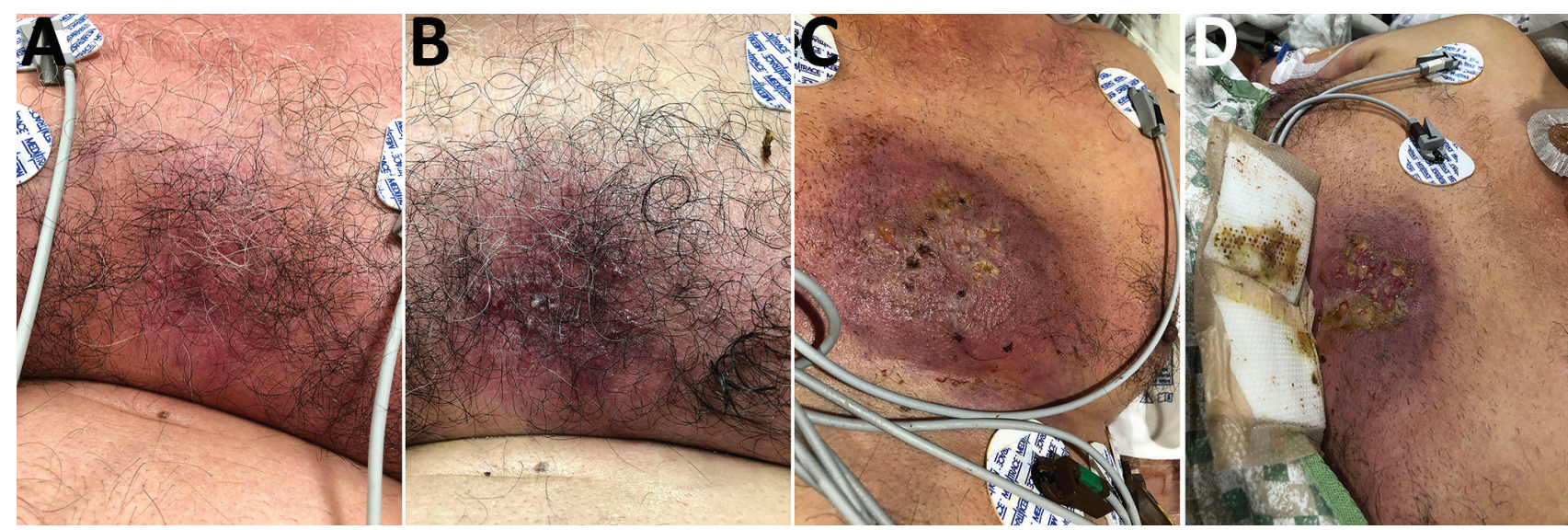

Figure 1. Progressive changes in a cutaneous chest wound for a 63-year-old man who had melioidosis, Texas, USA, 2018. Images were obtained on A) day 3, B) day 4, C) day 9, and D) day 10 after his initial visit to hospital A on November 17, 2018.

Because the isolate had an unremarkable resistance profile and B. pseudomallei was not specifically listed on the Texas Notifiable Conditions List, the automated system at hospital B did not generate an alert indicating it required LRN confirmation, and the isolate was not promptly reported to Texas Department of State Health Services (DSHS). On November 27, Houston LRN reported to DSHS Region 8 (San Antonio, TX, USA) confirmation of the isolate from hospital A as B. pseudomallei. The Houston LRN and hospital A destroyed the remaining isolates because of regulations surrounding handling of select agents.

DSHS and CDC collaboratively investigated the source of the patient's exposure to B. pseudomallei and performed risk assessments to identify potential laboratory exposures at both hospitals.

The patient's only reported travel outside Texas was a visit to Monterrey, Mexico, 30 years before illness

\begin{tabular}{|c|c|c|}
\hline Drug & $\mathrm{MIC}, \mu \mathrm{g} / \mathrm{mL}$ & Result \\
\hline Amikacin & $>32$ & $\mathrm{R}$ \\
\hline Ampicillin & $>16$ & $\mathrm{R}$ \\
\hline Ampicillin/sulbactam & $>16 / 8$ & $\mathrm{R}$ \\
\hline Aztreonam & $>16$ & $\mathrm{R}$ \\
\hline Cefazolin & $>16$ & $\mathrm{R}$ \\
\hline Cefepime & $>16$ & $\mathrm{R}$ \\
\hline Cefotaxime & 32 & $\mathrm{R}$ \\
\hline Cefoxitin & $>16$ & $\mathrm{R}$ \\
\hline Ceftazidime & 8 & $\mathrm{~S}$ \\
\hline Ceftriaxone & $>32$ & $\mathrm{R}$ \\
\hline Cefuroxime & $>16$ & $\mathrm{R}$ \\
\hline Ciprofloxacin & $>2$ & $\mathrm{R}$ \\
\hline Gentamicin & $>\overline{8}$ & $\mathrm{R}$ \\
\hline Ertapenem & $>1$ & $\mathrm{R}$ \\
\hline Meropenem & $\leq 1$ & $S$ \\
\hline Piperacillin/tazobactam & $<16$ & $S$ \\
\hline Tobramycin & $>8$ & $\mathrm{R}$ \\
\hline Trimethoprim/sulfamethoxazole & $<2 / 38$ & $\mathrm{~S}$ \\
\hline
\end{tabular}

${ }^{*} \mathrm{R}$, resistant; S, susceptible. onset. Before becoming ill, he resided on a small rural ranch without running water from a municipal source or private well. He purchased water for nondrinking use from a local chlorinated municipal water utility. He used a 500-gallon uncovered tank to store the water and then pumped the water into a 1,600-gallon storage tank. He cleaned the tank 1-2 times/month by climbing inside to scrub the walls. The last cleaning was 2 days before illness onset.

During environmental sampling in December 2018, we collected 56 specimens from the patient's home and property. We concentrated large-volume (30-190 L) water samples on site and processed as described (8). We collected soil samples $(50 \mathrm{~mL})$ at a depth of $30 \mathrm{~cm} \mathrm{(9)} \mathrm{from} 8$ sites in moist soil. Other environmental samples included surface swabs $(10 \times$ 10 inch) of the inside of both water storage tanks and swabs of indoor and outdoor plumbing fixtures. We processed specimens by using international guidelines (9) and tested for presence of B. pseudomallei by using culture and real-time quantitative PCR (10). All specimens were negative for $B$. pseudomallei.

We identified 7 laboratory personnel at hospitals $\mathrm{A}$ and $\mathrm{B}$ who had low-risk exposures to the $B$. pseudomallei isolates (5). Recommendations included temperature monitoring twice a day for 21 days and collecting serum samples at 1, 2, 4 and 6 weeks postexposure (5). We performed serologic testing by using an indirect hemagglutination assay (11). All samples were negative for $B$. pseudomallei antibodies (cutoff value 1:40).

Hospital B submitted the patient's blood culture isolate (TX2018b) for whole-genome sequencing (National Center for Biotechnology BioProject no. PRJNA545616). Multilocus sequence typing identified the isolate as sequence type 297 (12). The 8 other sequence type 297 isolates are all associated with North 


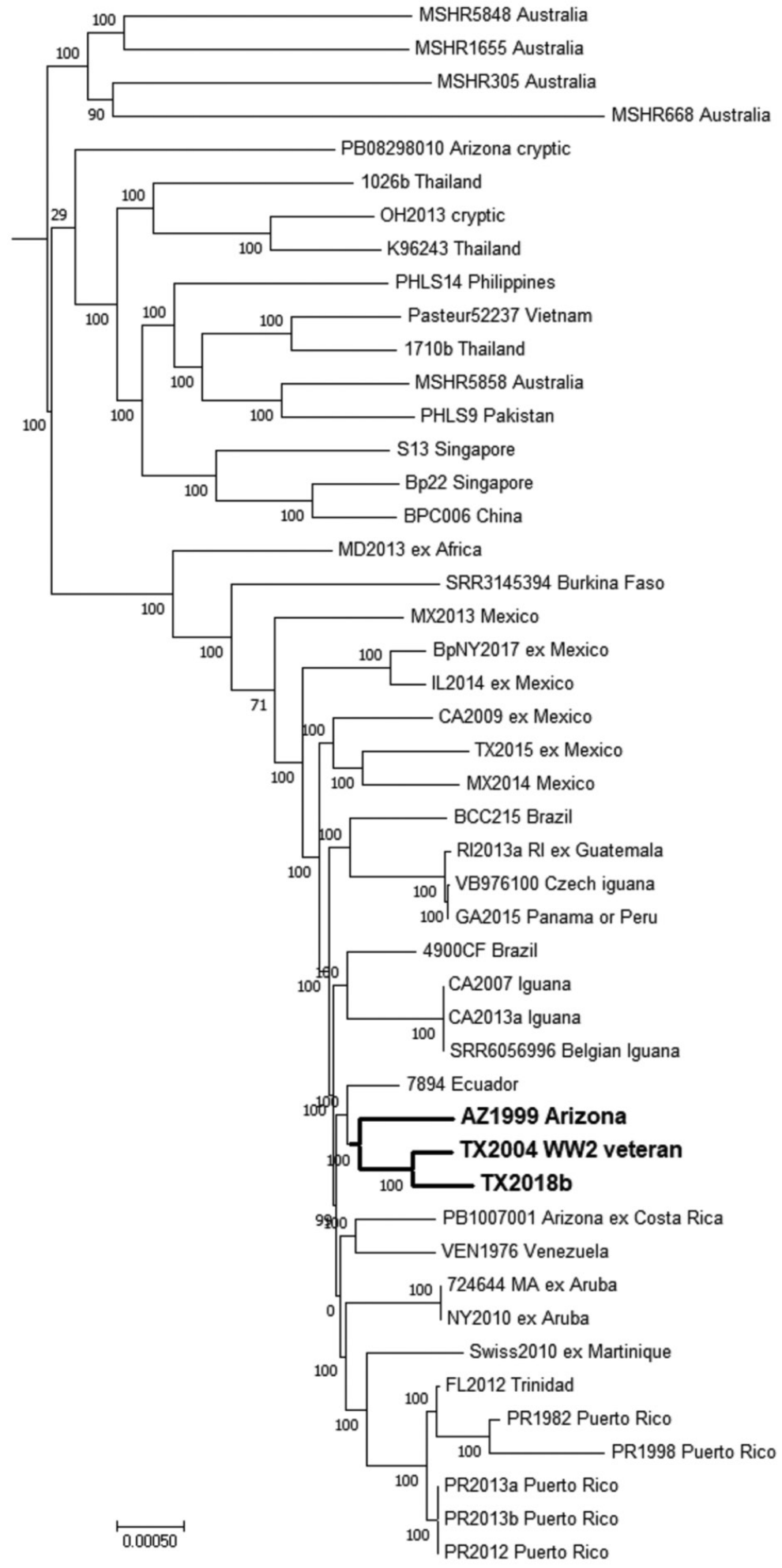

Figure 2. Dendrogram for characterization of Burkholderia pseudomallei isolate TX2018b from a 63-year-old man in Texas, USA, by comparison with reference genomes. Maximum-parsimony phylogenetic analysis based on core single-nucleotide polymorphisms (SNP) by using Parsnp, a component of the Harvest 1.3 software (https://github.com/marbl/harvest). Bold indicates clusters of genomes associated with the southwestern United States; the 2004 patient resided in the same county as the patient described in this article. Numbers at each node are bootstrap percentages. Scale bar indicates nucleotide substitutions per SNP. 
America (1). A higher resolution single-nucleotide polymorphism comparison of the draft genome assembly with other public assemblies containing geographic data also indicated that the isolate is associated with others from North America. TX2018b grouped closest to TX2004 and to a lesser extent to AZ1999 (BioProject no. PRJNA545640) (Figure 2).

TX2004 was isolated in 2004 from a patient residing in the same Texas county as our patient (13). It was originally hypothesized that the 2004 patient was infected 62 years before disease onset, while serving during World War II in Southeast Asia (13). However, TX2004 is not related to strains from Southeast Asia but to strains from the Americas (1). Other regional travel by the 2004 patient was not reported. AZ1999 was isolated from a patient treated in 1999 in Arizona who had recently emigrated from El Salvador. Where exposure occurred for that patient is unknown, but that isolate groups closer to TX2004 and TX2018b than to other examples associated with Central America (Figure 2).

\section{Conclusions}

The source of this patient's infection remains unknown. However, genomic analysis showed that the patient isolate groups with existing isolates collected from other patients in the southwestern United States. Isolates TX2004 and TX2018b were collected $\approx 15$ years apart from patients living in the same Texas county at time of illness onset and group together, a finding that suggests B. pseudomallei might be present in the environment in this area. Furthermore, these 2 isolates might represent a new clade endemic to the continental United States. Further investigation is warranted because this region is predicted to have suitable habitats for B. pseudomallei (14).

These findings call into question possible reactivation of melioidosis decades after travel to melioidosis-endemic regions. Instead of a 62-year incubation period, the patient infected with TX2004 might have had an unknown local environmental exposure that preceded symptom onset. A 1991 case report of an 18-year latency for a Vietnam War veteran indicated that this patient was also living in the southwestern United States (New Mexico) at time of symptom onset and had not traveled outside the continental United States since 1971 (15). On the other hand, although geographic and genotypic links between the 2 Texas cases suggest a local source, B. pseudomallei exposure for the patient infected with TX2018b could have occurred 30 years earlier, while visiting Mexico, and the patient infected with TX2004 might have had unreported regional travel before illness onset. Only when $B$. pseudomalle $i$ is isolated from the environment can it be definitively stated that B. pseudomallei is endemic to the continental United States.

B. pseudomallei infection should be included in a differential diagnosis for a patient with compatible disease, even without reported travel history. Increased awareness among healthcare workers and diagnostic laboratory personnel for melioidosis as a disease potentially endemic to the southwestern United States is critical to improve case outcomes and prevent laboratory exposures.

In addition, melioidosis is caused by a Tier 1 overlap Select Agent, but reporting of cases to CDC is voluntary $(2,7)$. Making melioidosis nationally notifiable could improve surveillance and recognition and clarify distribution and potential sources of B. pseudomallei infection in the United States.

\section{Acknowledgments}

We thank the patient and his family for providing photographs of the patient's progressing cutaneous chest wound and the Arizona Department of Health and Maricopa County Department of Health for providing background information on strain AZ1999.

\section{About the Author}

Dr. Cossaboom is a veterinary epidemiologist in the Division of High-Consequence Pathogens and Pathology, National Center for Emerging and Zoonotic Infectious Diseases, Centers for Disease Control and Prevention, Atlanta, GA. Her primary research interests include epidemiology of and outbreak response for zoonotic diseases of public health importance.

\section{References}

1. Gee JE, Gulvik CA, Elrod MG, Batra D, Rowe LA, Sheth M, et al. Phylogeography of Burkholderia pseudomallei isolates, Western Hemisphere. Emerg Infect Dis. 2017;23:1133-8. https://doi.org/10.3201/eid2307.161978

2. Benoit TJ, Blaney DD, Doker TJ, Gee JE, Elrod MG, Rolim DB, et al. A Review of melioidosis cases in the Americas. Am J Trop Med Hyg. 2015;93:1134-9. https://doi.org/10.4269/ ajtmh.15-0405

3. Wiersinga WJ, Virk HS, Torres AG, Currie BJ, Peacock SJ, Dance DA, et al. Melioidosis. Nat Rev Dis Primers. 2018;4:17107. https://doi.org/10.1038/nrdp.2017.107

4. Currie BJ. Melioidosis: evolving concepts in epidemiology, pathogenesis, and treatment. Semin Respir Crit Care Med. 2015;36:111-25. https://doi.org/10.1055/s-0034-1398389

5. Peacock SJ, Schweizer HP, Dance DA, Smith TL, Gee JE, Wuthiekanun V, et al. Management of accidental laboratory exposure to Burkholderia pseudomallei and B. mallei. Emerg Infect Dis. 2008;14:e2. https:/ / doi.org/10.3201/eid1407.071501

6. Federal Select Agent Program, 2017 [cited 2019 Apr 21]. http://www.selectagents.gov 
7. Adams DA, Thomas KR, Jajosky RA, Foster L, Baroi G, Sharp P, et al.; Nationally Notifiable Infectious Conditions Group. Summary of notifiable infectious diseases and conditions - United States, 2015. MMWR Morb Mortal Wkly Rep. 2017;64:1-143. https://doi.org/10.15585/

mmwr.mm6453a1

8. Mull B, Hill VR. Recovery of diverse microbes in high turbidity surface water samples using dead-end ultrafiltration. J Microbiol Methods. 2012;91:429-33. https:/ / doi.org/10.1016/j.mimet.2012.10.001

9. Limmathurotsakul D, Dance DA, Wuthiekanun V, Kaestli M, Mayo M, Warner J, et al. Systematic review and consensus guidelines for environmental sampling of Burkholderia pseudomallei. PLoS Negl Trop Dis.2013;7:e2105. https://doi.org/10.1371/journal.pntd.0002105

10. Novak RT, Glass MB, Gee JE, Gal D, Mayo MJ, Currie BJ, et al. Development and evaluation of a real-time PCR assay targeting the type III secretion system of Burkholderia pseudomallei. J Clin Microbiol. 2006;44:85-90. https:/ / doi.org/ 10.1128/JCM.44.1.85-90.2006

11. Alexander AD, Huxsoll DL, Warner AR Jr, Shepler V, Dorsey A. Serological diagnosis of human melioidosis with indirect hemagglutination and complement fixation tests. Appl Microbiol. 1970;20:825-33. https:/ / doi.org/10.1128/ AEM.20.5.825-833.1970
12. Godoy D, Randle G, Simpson AJ, Aanensen DM, Pitt TL, Kinoshita R, et al. Multilocus sequence typing and evolutionary relationships among the causative agents of melioidosis and glanders, Burkholderia pseudomallei and Burkholderia mallei. J Clin Microbiol. 2003;41:2068-79.

https:// doi.org/10.1128/JCM.41.5.2068-2079.2003

13. Ngauy V, Lemeshev Y, Sadkowski L, Crawford G. Cutaneous melioidosis in a man who was taken as a prisoner of war by the Japanese during World War II. J Clin Microbiol. 2005;43:970-2. https:/ / doi.org/10.1128/ JCM.43.2.970-972.2005

14. Limmathurotsakul D, Golding N, Dance DA, Messina JP, Pigott DM, Moyes CL, et al. Predicted global distribution of Burkholderia pseudomallei and burden of melioidosis. Nat Microbiol. 2016;1:15008. https:/ / doi.org/10.1038/ nmicrobiol.2015.8

15. Koponen MA, Zlock D, Palmer DL, Merlin TL. Melioidosis. Forgotten, but not gone! Arch Intern Med. 1991;151:605-8. https:/ / doi.org/10.1001/archinte.1991.00400030135027

Address for correspondence: Caitlin M. Cossaboom, Centers for Disease Control and Prevention, 1600 Clifton Rd NE, Mailstop H24-12; Atlanta, GA 30329-4027, USA; email: nrm9@cdc.gov

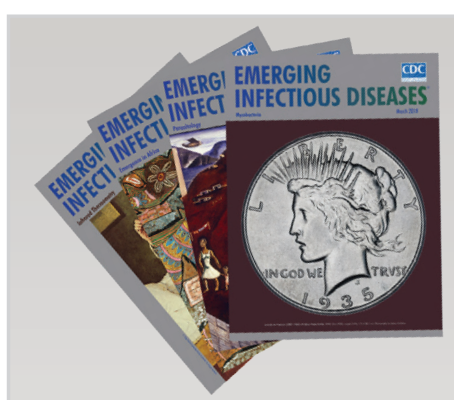

- Coccidioidomycosis Outbreaks, United States and Worldwide, 1940-2015

- Multistate Epidemiology of Histoplasmosis, United States, 2011-2014

- Epidemiology of Recurrent Hand, Foot and Mouth Disease, China, 2008-2015

- Capsule Typing of Haemophilus influenzae by Matrix-Assisted Laser Desorption/Ionization Time-of-Flight Mass Spectrometry

- Emergence of Streptococcus pneumoniae Serotype $12 \mathrm{~F}$ after Sequential Introduction of 7- and 13-Valent Vaccines, Israel

- Major Threat to Malaria Control Programs by Plasmodium falciparum Lacking Histidine-Rich Protein 2, Eritrea

- Use of Influenza Risk Assessment Tool for Prepandemic Preparedness

- Use of Verbal Autopsy to Determine Underlying Cause of Death during Treatment of Multidrug-Resistant Tuberculosis, India

- Seroprevalence of Dengue and Chikungunya Virus Antibodies, French Polynesia, 2014-2015
March 2018

- Increasing Prevalence of Nontuberculous Mycobacteria in Respiratory Specimens from US-Affiliated Pacific Island Jurisdictions

- Use of Genome Sequencing to Define Institutional Influenza Outbreaks, Toronto, Ontario, Canada, 2014-15

- Influenza Vaccination and Incident Tuberculosis among Elderly Persons, Taiwan

- Epidemiology and Molecular Identification and Characterization of Mycoplasma pneumoniae, South Africa, 2012-2015

- Prospective Observational Study of Incidence and Preventable Burden of Childhood Tuberculosis, Kenya

- Acquired Resistance to Antituberculosis Drugs in England, Wales, and Northern Ireland, 2000-2015

- Characteristics Associated with Negative Interferon- $\gamma$ Release Assay Results in Culture-Confirmed Tuberculosis Patients, Texas, USA, 2013-2015

- Genetic Spatiotemporal Anatomy of Plasmodium vivax Malaria Episodes in Greece, 2009-2013

\section{https://wwwnc.cdc.gov/eid/articles/issue/24/3/table-of-contents}

